\title{
Systematic review of the effectiveness of the Wilbarger protocol with children
}

This article was published in the following Dove Press journal:

Pediatric Health, Medicine and Therapeutics

30 November 2012

Number of times this article has been viewed

\section{Scott Weeks \\ Kobie Boshoff \\ Hugh Stewart}

School of Health Sciences Occupational Therapy Program, University of South Australia,

Adelaide, South Australia, Australia
Correspondence: Kobie Boshoff University of South Australia, School of Health Sciences, GPO Box 247I, Adelaide, SA 500I, Australia

Tel +6I 883021089

Fax +6I 883022645

Email kobie.boshoff@unisa.edu.au
Background: Sensory processing disorders have an estimated prevalence of 5\%-10\% in children without disability and $40 \%-88 \%$ in children with disability. A subtype of sensory processing disorder is sensory overresponsivity, which can result in fear, irritability, aggression, or avoidance behaviors in children. The Wilbarger protocol is the most prescriptive program used to treat sensory overresponsivity in children aged 2-12 years. Strong anecdotal evidence suggests that the Wilbarger protocol successfully reduces challenging behavior in children with sensory overresponsivity. The aim of this systematic review was to identify and appraise the existing evidence for the effectiveness of the Wilbarger protocol with children aged 0-18 years.

Methods: A systematic review was conducted of the peer-reviewed literature written in English. The electronic databases searched up to April 2012 included CINAHL, Ovid Medline (R), Embase, Scopus, the Cochrane Library, AMED, and the Web of Science. OT Seeker and Google Scholar were searched for missed literature, along with hand-searching of retained articles. Adult studies were excluded. The Critical Review Form for Quantitative Studies by McMaster University and the levels of hierarchy from the Australian National Health and Medical Research Council were used to appraise the literature.

Results: A total of 341 studies were found, and 302 were screened for eligibility after duplicates were removed. Four level IV intervention (case series with pretest/posttest) studies were included for indepth review. All four studies had very small sample sizes, exhibited low methodological quality, differed in outcome measures used, and lacked homogeneity of samples and treatment fidelity.

Conclusion: A lack of high quality evidence currently exists to support or refute the use of the Wilbarger protocol with children. While the grade of recommendation, as proposed by the Australian National Health and Medical Research Council, suggests that the Wilbarger protocol should be applied with caution, emerging evidence from these studies warrants future robust research on this topic. Clinicians are advised to use clear outcome measures when using the Wilbarger protocol with clients.

Keywords: Wilbarger protocol, pediatrics, allied health

\section{Introduction}

One of the most extensively used approaches by pediatric occupational therapists is the sensory integration approach. ${ }^{1}$ It is used as a frame of reference by occupational therapists to guide their clinical reasoning and practice when working with children who have sensory integration and processing difficulties. ${ }^{1}$ Children with sensory integration and processing difficulties experience decreases in occupational performance, most notably in activities of daily living, instrumental activities of daily living, school, 
and social participation. ${ }^{2}$ Evidence for the effectiveness of occupational therapy using a sensory integration approach is inconclusive, even though numerous outcome studies exist. Supporters and critics are equally as passionate with their arguments concerning this controversial approach, often arising in heated debate. ${ }^{3}$ The most recent example to cause such debate is a policy statement by the American Academy of Pediatrics. ${ }^{4}$ In the occupational therapy literature, the terms "sensory integration" and "sensory processing" are used interchangeably and often with controversy. ${ }^{5}$

The term "sensory integration" lacks consistency between disciplines with regard to its definition. For instance, in neuroscience, the term refers to a cellular process where the signals from two or more senses combine in the central nervous system. ${ }^{5}$ On the other hand, sensory integration as defined by Ayres in 1972, and used by occupational therapists to guide their theoretical practice, is a neurological process of "receiving, registering, modulating, organizing, and interpreting" sensory input, ${ }^{6}$ in order to adapt to situational demands and produce functional outcomes. ${ }^{7}$ Furthermore, sensory integration is also used by occupational therapists to explain assessment and intervention. ${ }^{8}$ In the more recent occupational therapy literature, sensory processing is a term which now encompasses management of the peripheral and central nervous systems, with regards to reception, modulation, integration, and organization of sensory input, as well as the behavioral responses to this input. Sensory integration is the capacity of the central nervous system to process this sensory input, and hence only one component of sensory processing. ${ }^{9}$

The diagnosis of sensory integration dysfunction was based on Ayres' hypothesis that some children experience challenges concerning purposeful behaviors due to sensory integration impairment. ${ }^{6}$ Recently, it has been proposed that this diagnosis be renamed "sensory processing disorder" if daily routines are impaired by sensory processing difficulties. ${ }^{8}$ As a result, this proposed nosology allows the terminology for the disorder to be distinguished from the terminology used for theory, intervention, and cellular processes. Sensory processing disorder adversely affects motor, cognitive, behavioral, and emotional development, and is estimated to be prevalent in 5\%-10\% of children without disability and $40 \%-88 \%$ of children with disability. ${ }^{10}$ Further subtypes of sensory processing disorder have been proposed to allow greater opportunity for homogenous samples in empirical research and more targeted intervention approaches. ${ }^{8}$

One subtype of sensory processing disorder is sensory overresponsivity. ${ }^{8}$ Sensory overresponsivity is defined as a person responding faster, more intensely, or for extended duration to sensation, in comparison with a person who has typical responses to sensation. ${ }^{8}$ Wilbarger and Wilbarger in 1991 had originally named this phenomenon "sensory defensiveness" and described it as a "tendency to react negatively or with alarm to sensory input which is generally considered harmless or non-irritating." 11 Sensory overresponsivity can result in fear, irritability, aggression, or avoidance behaviors for children. ${ }^{12}$ It has recently been established that $16.5 \%$ of children aged 7-11 years in the United States ${ }^{13}$ and 15\% of children aged 3-10 years in Israe ${ }^{14}$ experience sensory overresponsivity.

Parents of children who are overresponsive to sensation often seek intervention for their children from occupational therapists. The Wilbarger protocol is the most prescriptive ${ }^{15}$ and popular program used to treat sensory overresponsivity in children aged 2-12 years. ${ }^{16,17}$ No current statistical data are available regarding the prevalence of the use of the Wilbarger protocol, except for an unpublished master's thesis written in 2001 , which states that $78 \%$ of 64 American pediatric occupational therapists surveyed reported using the Wilbarger protocol as part of their rehabilitative modalities. ${ }^{18}$ Additionally, it was highlighted in 2007 that Avanti Educational Programs reported that 20,000 Therapressure ${ }^{\mathrm{TM}}$ brushes were ordered per annum and specialized training had been provided to over 15,000 health professionals worldwide. ${ }^{15}$

The Wilbarger protocol involves three prescribed components. Firstly, the body is brushed with a specifically manufactured, nonscratching (Therapressure ${ }^{\mathrm{TM}}$ ) brush to the hands, arms, back, legs, and feet. A prescribed brushing technique utilizing deep pressure is advised to avoid noxious stimuli (ie, scratching or tickling). This is followed by compressions to the major joints to stimulate proprioception and completed with a prescribed set of sensorimotor activities named the "sensory diet." Parents are trained by therapists with specialized Wilbarger protocol training and are required to apply this technique accurately and repeatedly every 90-120 minutes per day, for a minimum of 2 weeks. ${ }^{15,16,19}$ Parents and therapists have provided strong, subjective anecdotal evidence that the Wilbarger protocol successfully reduces behavioral responses to environmental stimuli in children displaying sensory overresponsivity. ${ }^{15}$

In 2003, an evidence review called for research to be produced to test the effectiveness of the Wilbarger protocol, because evidence that was published and objective was scant. ${ }^{17}$ Of note, only $2.6 \%$ of the American pediatric occupational therapists who reported using the Wilbarger protocol expressed concerns with the lack of empirical evidence. ${ }^{18}$ One 
explanation may be due to the strong anecdotal evidence that exists. However, therapists are unable to rely solely on their theoretical viewpoints and clinical experiences in a current health care context that promotes the use of practice informed by research evidence. ${ }^{20}$ The aim of this systematic review was to identify and appraise the evidence that sought to test the effectiveness of the Wilbarger protocol with children aged 0-18 years.

\section{Methods}

\section{Search strategy}

A systematic literature search was conducted to identify peer-reviewed publications that addressed the effectiveness of the Wilbarger protocol. The electronic databases searched up to April 2012 included CINAHL, Ovid Medline (R, 1948 to present), Embase (1974 to present), Scopus, the Cochrane Library, AMED, ERIC, Proquest, PsychINFO, and the Web of Science. OT Seeker, Google Scholar, and reference lists of retained articles were searched further for missed literature.

The authors were aware that limited peer-reviewed articles existed and used only the "intervention" component of PICO (patient, intervention, comparison, outcome) to ensure that all articles were found. The "intervention" was specified as the Wilbarger protocol. Search terms included "Wilbarger" OR "Wilbarger protocol" OR "Wilbarger brushing" OR "Therapressure” OR "deep pressure and proprioceptive technique" OR "sensory summation technique". Adult studies were excluded when the full text articles were screened and no comparison group or outcome variables were specified. No date limits were placed on the search.

\section{Inclusion and exclusion criteria}

Once all articles had been identified, studies were included in the critical appraisal process if they were published in peer-reviewed journals, were written in English, were retrievable through the UniSA library, and primarily discussed or demonstrated the effectiveness of the Wilbarger protocol (or a component of the protocol). Studies were excluded if the effects of the Wilbarger protocol were studied in adults, if it was a literature synthesis, or if the intervention comprised multiple sensory integration techniques, where the amount of Wilbarger protocol administered could not be clearly defined. Due to the paucity of literature available on this topic, no studies were excluded on the basis of study design. Peer-reviewed conference abstracts/conference slides, gray literature, and studies reporting the perceived effectiveness of the intervention were discussed under a separate section to represent the total body of evidence available on this topic. Two reviewers (SW and KB) were involved in the article selection process, and full agreement was reached on the included articles.

\section{Critical appraisal process}

The critical appraisal tool known as the Critical Review Form for Quantitative Studies from McMaster University was used by SW to review the methodological quality and summarize the findings of the retained papers. ${ }^{21}$ This tool was selected by the authors as they deemed it to be most appropriate for the small number of studies included and the difference in outcome measures. Additionally, this tool provides a quality rating that allocates a total numerical score of 15 to rank each study. Answers of "yes" are awarded one point, whereas answers of "no," "not addressed," and "N/A" are awarded zero points. The answer "not addressed" has also been interpreted as not providing sufficient information to award a point clearly. The results were assessed and verified by KB for accuracy, and consensus was reached on all four studies.

The Australian National Health and Medical Research Council's evidence hierarchy was used to assign a level of evidence to each study included. ${ }^{22}$ The Australian National Health and Medical Research Council's body of evidence matrix was used to interpret the findings of the studies included to provide an evidence-based recommendation. ${ }^{22}$ The body of evidence matrix comprises five components. The first two, ie, evidence base and consistency, refer to the internal validity of the studies. The third appraises the clinical impact of the studies, whereas the fourth addresses the generalizability of findings to the wider population. The fifth, applicability to the Australian (or local) health care system, has been omitted because this review was intended for an international audience.

\section{Results \\ Search findings}

The original search using the above search strategy identified 337 studies through database searching and four studies through hand-searching, which were reviewed for duplicates. A total of 302 studies were excluded on the basis of screening titles and abstracts. Abstracts were excluded during the screening process if they described the theory of the Wilbarger protocol rather than testing its effectiveness, or were irrelevant, such as the term "Wilbarger" representing an author, place, or topic not related to the Wilbarger protocol. The remaining 16 studies were read in full for eligibility, leaving four 
peer-reviewed studies which met the inclusion criteria and were able to be included in the critical appraisal of this systematic review. A further eight studies that did not meet the inclusion/exclusion criteria, but addressed the effectiveness of the intervention, are discussed in the section entitled "Other literature." Figure 1 illustrates the literature selection process according to the PRISMA 2009 flow diagram. ${ }^{23}$

\section{Study range and characteristics}

Table 1 provides a summary of the design characteristics of the studies included. All four studies that met the inclusion criteria were case series with pretest/posttest outcomes. This is considered the lowest level of evidence (level IV intervention evidence) according to the Australian National Health and Medical Research Council's evidence hierarchy. ${ }^{22}$ Three of the studies analyzed their results by using subjects serving as their own controls, ${ }^{15,16,24}$ whilst the fourth also compared the results of one participant receiving Wilbarger protocol against a control receiving a nonspecific child-guided brushing technique. ${ }^{25}$ Three of the studies were undertaken in the United States $^{15,16,25}$ and one in Australia. ${ }^{24}$

The sample sizes ranged from one to four participants, indicating very small sample sizes overall. No justification was provided in any study for the size of the sample. All four studies were limited to boys aged 3-5 years. All used convenience sampling from local occupational therapy clinics as a recruiting method. In the study where one participant was compared with a control, the participants were randomized to treatment and control interventions, but the assessor was not blinded to group allocation. ${ }^{25}$

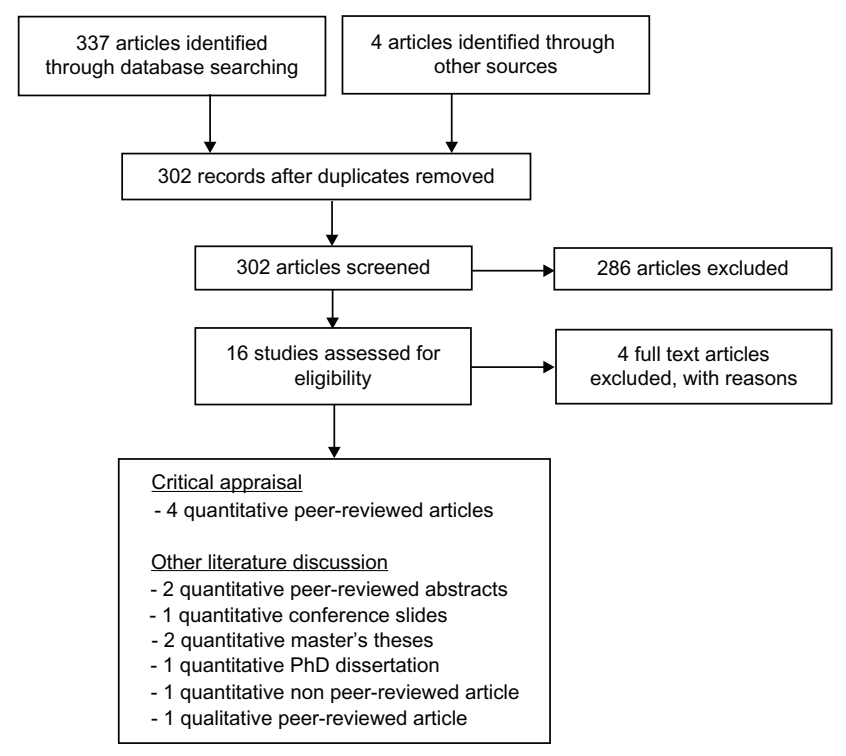

Figure I Flow chart of the literature selection process.
Three of the four studies used sensory overresponsivity (sensory defensiveness) as the primary diagnosis. ${ }^{15,24,25}$ One study made the diagnosis on the basis of parents completing a nonstandardized sensory checklist and from the observations of an occupational therapist, ${ }^{24}$ another on the basis of behaviors consistent with sensory modulation disorder as identified by the treating occupational therapist, ${ }^{25}$ and the third used the Short Sensory Profile to confirm the diagnosis made by an occupational therapist. ${ }^{15}$ The samples were not homogenous, as evidenced by one study that included participants with combinations of overresponsive and underresponsive behaviors, ${ }^{15}$ and another that compared results from a child with autism spectrum disorder with those from a child with pervasive developmental delay not otherwise specified. ${ }^{25}$ The fourth study focused on reducing stereotyped behaviors associated with autism, ${ }^{16}$ for which the Wilbarger protocol was not designed.

There was a lack of consistency between studies with regard to outcome measures (see Table 1). Three studies included a standardized assessment as a part of their outcome measures. ${ }^{15,24,25}$ These assessments include the School Function Assessment, ${ }^{25}$ Short Sensory Profile, Conner's Rating Scales Revised, ${ }^{15}$ and the Miller Assessment for Preschoolers. ${ }^{24}$ Only two of these studies claimed acceptable psychometric properties for their outcome measures, which included the School Function Assessment, ${ }^{25}$ salivary cortisol, Short Sensory Profile, and Conner's Rating Scales Revised. ${ }^{15}$ One study that used observations in 10 -second intervals to collect stereotypy data aimed to increase its rigor by calculating interobserver agreement. The interobserver agreement reported by the two researchers collecting data was $97 \%$ for the pretest/posttest measurements and $96 \%$ for measurements made during intervention. ${ }^{16}$

\section{Fidelity of intervention delivery}

All four studies implemented variations of the Wilbarger protocol. A study by Davis et al prescribed the frequency and duration of brushing within the Wilbarger protocol guidelines at approximately seven times per day, with evenly spaced intervals, for 6 weeks. However, it was not clearly stated if the child received joint compressions or a sensory diet. The child's occupational therapist provided hands-on training to the mother, who administered most of the brushing. In turn, the mother provided hands-on training to the in-home behavioral therapist, who administered brushing when the mother was unavailable. The authors stated that treatment fidelity data were not collected and, therefore, the extent to which the protocol was adhered to was unknown. ${ }^{16}$ 
Benson et al appeared to remain close to the Wilbarger protocol prescription by reporting that the participant randomized to the Wilbarger protocol intervention received brushing three times during school hours (at 9 am, $11 \mathrm{am}$, and $1 \mathrm{pm}$ ) and a consistent schedule by parents at home. The time interval between brushing at home and administration of joint compressions as a part of the intervention was not stated, making it difficult to assess treatment fidelity. The brushing protocol was administered in conjunction with a sensory diet at school and home. Intervention lasted for 21 days. ${ }^{25}$

Stagnitti et al reported that the child in their study received less frequent intervention per day than that recommended in the Wilbarger protocol. Brushing and joint compressions were administered three times per day in the first week and 4-5 times per day in the second week of intervention due to parental commitments. A sensory diet was recommended to the family during this period, although it was not stated explicitly if this was adhered to. The participant attended four occupational therapy sessions on completion of the Wilbarger protocol, where only equipment was mentioned. At 5 months after the initial assessment, a repeat of the Wilbarger protocol was administered three times per day for 2 weeks; however, this was implemented in conjunction with behavioral and narrative therapy techniques. ${ }^{24}$

Kimball et al modified the Wilbarger protocol by administering a single application of Wilbarger protocol to each participant, once per week for 4 weeks during scheduled occupational therapy sessions. It was not clearly stated in the methodology which components of the Wilbarger protocol were administered at each individual application, nor was it stated who administered the intervention to each participant. The authors did not clearly state if other interventions had occurred during the scheduled occupational therapy sessions. ${ }^{15}$

\section{Rating scale and body of evidence matrix}

Table 2 shows the rating scores for the studies included and Table 3 provides a summary of the results of the body of evidence matrix. The grade of recommendation as proposed by the Australian National Health and Medical Research Council was assessed as "level D."'22 This implies that evaluation of the published peer-reviewed evidence for the effectiveness of the Wilbarger protocol with children under the age of 18 years should be done with caution, because the body of evidence is weak.

\section{Discussion}

Due to variations in prescription of the intervention, the diversity of samples, and the difference in outcome measures used between studies, the results are reported individually rather than as a synthesis. In the study by Davis et al, administration of the Wilbarger protocol did not show any marked effects in terms of decreasing levels of stereotypy in a boy with autism. Baseline analysis showed that stereotypy behavior occurred in the boy at a mean of $40 \%$ of intervals (range 16\%-54\%) prior to intervention. Intervals of stereotypy increased to a mean of $52 \%$ (range $41 \%-63 \%$ ) during week 3 , and increased further to a mean of $55 \%$ (range $37 \%-69 \%$ ) during week 5 of intervention. Baseline measures were repeated 6 months after completion of the Wilbarger protocol (8 months from pretest to posttest baselines) showing a decrease of intervals of stereotypy to a mean score of $28 \%$ (range $17 \%-42 \%$ ). The authors concluded that the Wilbarger protocol did not reduce stereotypy during the brushing phase or show a "marked distinction between brushing and nonbrushing phases." 16

According to Benson et al, the effectiveness of brushing is not dependent on following a timed schedule. The participant with autism spectrum disorder who received the Wilbarger protocol intervention experienced most improvement in School Function Assessment scores for the categories of "following social conventions" and "behavior regulation." His improvement was $4 \%$ in both categories from pretest to posttest. The results were compared with those in a control participant with pervasive developmental delay not otherwise specified. This participant received nonspecified child-guided technique intervention and experienced the most improvements in "compliance with adult directives and school rules," "task behavior/completion," and "behavior regulation," with gains of $6 \%, 11 \%$, and $10 \%$, respectively. It was concluded that the intervention of brushing was more important in improving occupational performance than the time allocated between brushing intervals. Therefore, according to the authors, the protocol should be administered based on the child's needs and not according to the prescribed schedule. ${ }^{25}$

Following initial administration of the Wilbarger protocol in the case study by Stagnitti et al, the mother reported that the incidence of the participant's temper tantrums at kindergarten had decreased, his participation in groups had increased, he allowed others to touch him, his social participation had increased, he had been cautioned for the first time for risktaking behavior, and his hand-eye and foot-eye coordination (kicking a ball) had improved. An area that had seen less change was that he was still curling into a ball, although this was less frequent. At 6 months after the initial assessment, the participant showed improved Miller Assessment for Preschoolers scores (no areas of difficulty) and a marked improvement between before and after on the sensory checklist. His 


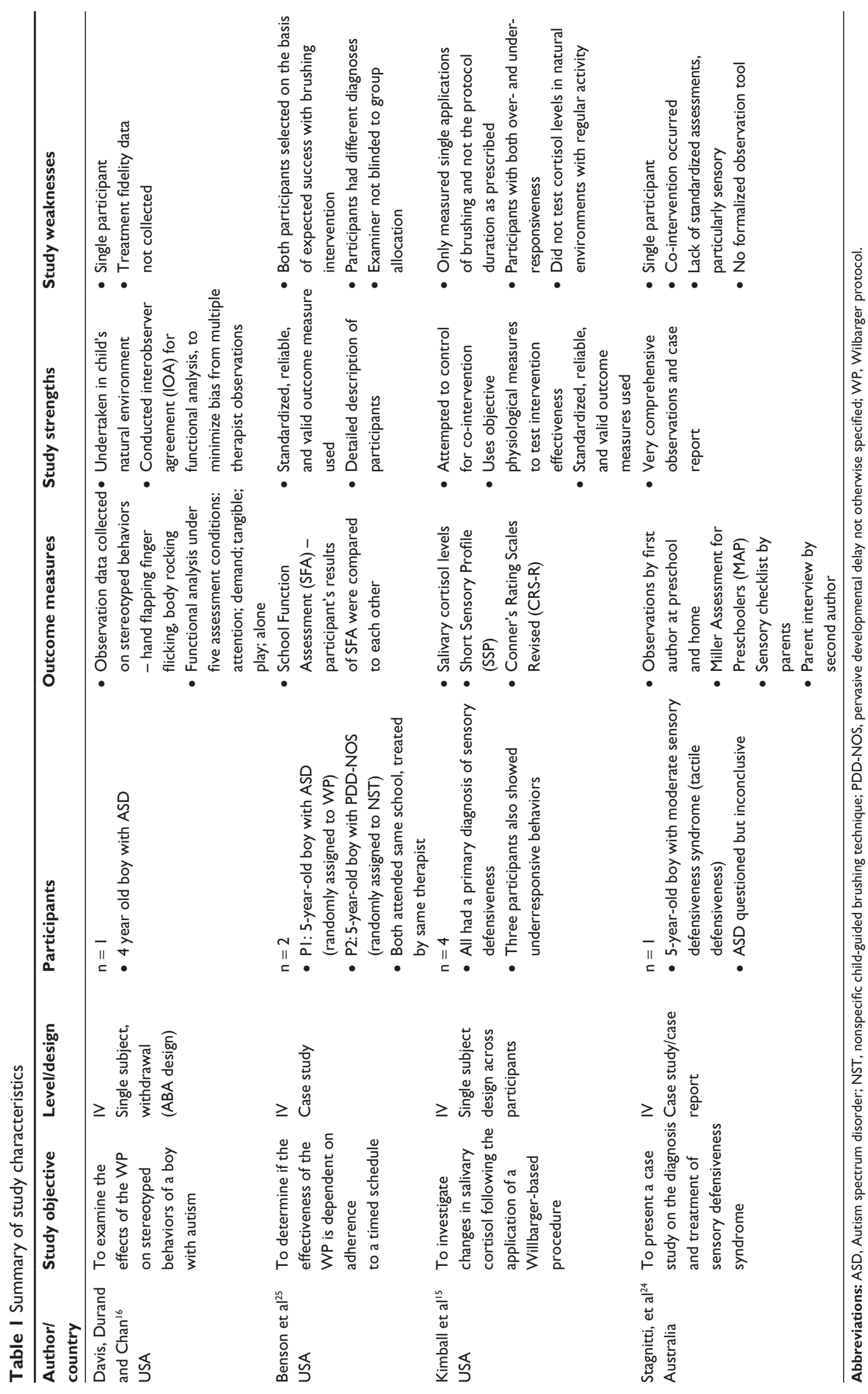


Table 2 McMaster rating scores (out of I5)

\begin{tabular}{|c|c|c|c|c|}
\hline & Davis et al $^{16}$ & Benson et $\mathrm{al}^{25}$ & Kimball et al ${ }^{15}$ & Stagnitti et $\mathrm{al}^{24}$ \\
\hline Was the purpose stated clearly? & Yes & Yes & Yes & Yes \\
\hline Was relevant background literature reviewed? & Yes & Yes & Yes & Yes \\
\hline Study design specified and appropriate? & Yes & Yes & Yes & Yes \\
\hline Was the sample described in detail? & Yes & Yes & Yes & Yes \\
\hline Was the sample size justified? & No & No & No & No \\
\hline Were the outcome measures reliable? & Not addressed & Yes & Yes & No \\
\hline Were the outcome measures valid? & Not addressed & Yes & Yes & No \\
\hline Intervention was described in detail? & Yes & Yes & Yes & Yes \\
\hline Contamination was avoided? & $N / A$ & No & $\mathrm{N} / \mathrm{A}$ & $N / A$ \\
\hline Co-intervention was avoided? & No & Not addressed & No & No \\
\hline Results reported for statistical significance? & No & No & No & No \\
\hline Were the analysis method(s) appropriate? & Yes & Yes & Yes & Yes \\
\hline Clinical importance was reported? & Yes (briefly) & Yes & Yes & Yes \\
\hline Drop-outs were reported? & No & No & No & No \\
\hline Conclusions were appropriate? & Yes & Yes & Yes & Yes \\
\hline Total score & 8 & 10 & 10 & 8 \\
\hline
\end{tabular}

behavior was observed to be jovial, he showed sociable behavior towards his occupational therapist and family members, and showed increased enthusiasm for rough-and-tumble play. At 9 months after the initial assessment, it was reported by his mother that "everything was going magnificently" and he was attending school. The authors concluded that the Wilbarger protocol was effective. ${ }^{24}$

In the study by Kimball et al, an association between single administrations of a Wilbarger-based procedure and modulation of cortisol levels in all four participants was identified. Participants 2 and 4 showed a decrease in cortisol levels from pretest to posttest for all four administrations of the intervention. Conversely, participant 3 showed an increase in cortisol levels for all four administrations. However, participant 1 showed a decrease in cortisol levels from pretest to posttest for the first three administrations and an increase on the fourth administration. The fourth test had shown pretest cortisol levels lower than the posttest levels for his first three administrations and the lowest of any participant in the study. The authors suggested that the boy was under-aroused at the beginning of testing and that intervention elevated him to a more "normal level," which is consistent with Wilbarger protocol theory. There were no changes in scores on the Short Sensory Profile or Conner's Rating Scale Revised over the 4 weeks of the study in any of the participants. The authors stated that they expected no change in these scores,

Table 3 NHMRC body of evidence matrix

\begin{tabular}{|c|c|c|}
\hline Component & Grade & Comments \\
\hline Evidence base & $\begin{array}{l}\text { D (poor) } \\
\text { Level IV studies, or level I to III } \\
\text { studies/SRs with a high risk of bias }\end{array}$ & $\begin{array}{l}\text { - Studies: }(n=4) \\
\text { - Low level of evidence: level IV (case series with pretest/posttest outcomes) } \\
\text { - No study calculated sample size estimates } \\
\text { - Unable to calculate statistical significance due to small sample sizes } \\
\text { - Low use of standardized assessments }\end{array}$ \\
\hline Consistency & $\begin{array}{l}\mathrm{D} \text { (poor) } \\
\text { Evidence is inconsistent }\end{array}$ & $\begin{array}{l}\text { - Vast differences in outcomes measures between studies } \\
\text { - Results differ across studies } \\
\text { - Samples lack homogeneity } \\
\text { - Lacking intervention fidelity between studies }\end{array}$ \\
\hline Clinical impact & $\begin{array}{l}\text { D (poor) } \\
\text { Slight or restricted }\end{array}$ & $\begin{array}{l}\text { - Only four studies with low level of evidence } \\
\text { - Intervention lacked homogeneity } \\
\text { - Co-intervention bias }\end{array}$ \\
\hline Generalizability & $\begin{array}{l}\text { D (poor) } \\
\text { Population/s studied in body of evidence differ to } \\
\text { target population and hard to judge whether } \\
\text { it is sensible to generalize to target population }\end{array}$ & $\begin{array}{l}\text { - Sample sizes too small } \\
\text { - Only boys studied } \\
\text { - Limited age range studied ( } 3-5 \text { yrs, } 10 \text { mo) }\end{array}$ \\
\hline $\begin{array}{l}\text { Grade of } \\
\text { recommendation }\end{array}$ & $\begin{array}{l}\text { D (weak/caution) } \\
\text { Body of evidence is weak and recommendation } \\
\text { must be applied with caution }\end{array}$ & $\begin{array}{l}\text { Poor methodological quality of studies, small sample sizes, most outcome } \\
\text { measures lacked psychometric properties and differed across studies, long-term } \\
\text { follow-up studies had high risk of bias through co-intervention and maturation }\end{array}$ \\
\hline
\end{tabular}

Abbreviations: NHMRC, National Health and Medical Research Council; SR, systematic review. 
because the intervention administered did not adhere strictly to Wilbarger protocol guidelines. On the other hand, it may imply that the intervention did not work, or that the outcome measures were not sensitive enough to detect change. The authors state that norms for salivary cortisol do not exist for children. $^{15}$

\section{Other literature}

Although the focus of this review was on full peer-reviewed studies, the following peer-reviewed conference abstracts/ slides, non-peer-reviewed studies, and studies of perceived effectiveness that were found during the search are discussed to cover the total body of evidence that exists for the Wilbarger protocol.

\section{Peer-reviewed conference abstracts/slides}

Three peer-reviewed conference abstracts/slides were found as a result of the systematic literature search. The first was reviewed from conference slides presented at the 43rd Australasian Society for the Study of Intellectual Disability Conference in $2008 .{ }^{26}$ The second and third were abstracts from the 24th Occupational Therapy Australia National Conference and Exhibition in 2011. 27,28 The author of the third abstract generously provided a copy of the master's thesis from which the abstract was generated.

The first was a randomized controlled study which allocated 16 children (11 boys and five girls) to a sensory protocol (experimental) group or a behavioral intervention (control) group. The participants were aged 4-10 years with a diagnosis of intellectual disability or developmental delay, present in two or more functional domains of sensory defensiveness, and requiring support for behavioral issues. The sensory protocol comprised the Wilbarger protocol and an individualized sensory diet as prescribed by the protocol. The duration of the intervention was not listed in the online conference slides. The Developmental Behaviour Checklist, Parent Sensory Protocol Diary, and four goal attainment scales were used as outcome measures, and all results were reported in terms of statistical significance. The results showed positive outcomes for both groups on parent-measured goals; however, the sensory group showed more positive outcomes than the behavioral group. Likewise, it was found that the sensory group showed more positive outcomes in behavioral goal attainment scores, although no difference was found between the groups for functional goal attainment scores. Additionally, there was no difference in total Developmental Behaviour Checklist scores between the two groups, except for the "anxiety" subscale where the sensory intervention was shown to reduce anxiety to a greater extent than the behavioral intervention. ${ }^{26}$

The same authors also undertook the study in the second abstract. The study objective was to determine if the sensory protocol improved functional and behavioral outcomes in children with severe sensory defensiveness. In this study, the age range was 6-12 years, the participant size increased to 30, and an extra outcome measure, ie, the Short Sensory Profile, was included. A randomized controlled crossover design was used, with concealed allocation. Again, the experimental group was the sensory (Wilbarger) protocol and the control group received behavioral support. Measurements were taken before and after two 6-week intervention phases. The results confirmed statistically significant differences between the groups with regard to functional and behavioral outcomes, in favor of the sensory protocol. ${ }^{27}$ These two studies appear to be the highest in methodological quality to be conducted on the Wilbarger protocol and hence would contribute greatly to the body of evidence if published in full in a peer-reviewed journal.

The third used a prospective multiple single-case approach, with a single subject design, to investigate the effectiveness of the Wilbarger protocol with children with sensory defensiveness. The participants were five boys, aged 3-4 years, who were eligible for early childhood intervention services. Four of these boys were diagnosed with autism spectrum disorder. The Wilbarger protocol was administered daily every 2 hours for 6 weeks with concomitant sensory diet activities three times per day. Treatment fidelity was a strength of this study. A statistically significant difference using paired-samples $t$-tests was shown, as well as a large effect size for goal attainment scores over the 6-week intervention. Likewise, a statistically significant difference was shown for three of the Sensory Profile quadrant scores, ie, the "sensitivity," "avoiding," and "seeking" categories. The highest $P$-values were for "sensitivity" and "avoiding," which are strong determinants of sensory overresponsivity and sensory defensiveness. This indicates that the children demonstrated a reduction in sensitive and avoiding behaviors following intervention. ${ }^{28}$

\section{Gray literature}

In an unpublished master's thesis, five boys and two girls with sensory integrative dysfunction (specifically sensory defensiveness) were observed on three types of engaging behaviors. The children were observed on visual, motor, and combined motor-visual engaging behaviors, for 5 minutes before and after brushing, to measure the short-term effects 
of the Wilbarger protocol. The author could not support the use of the Wilbarger protocol for a single application of brushing and joint compressions, but concluded that boys may respond better than girls to the intervention. ${ }^{29}$

Similarly, a PhD dissertation sought to explore the effects of the Wilbarger protocol on task engagement. In this study, a ten-year-old boy with autism spectrum disorder, who exhibited difficulty focusing on tasks, was assessed across multiple settings. The child was observed for five intervention sessions in a setting before being observed in the next setting. The child showed a $9 \%$ increase in task engagement in the computer laboratory setting, followed by a $19 \%$ improvement in the physical education setting and a $45 \%$ improvement in the general classroom setting. It was established that the setting determined the effectiveness of the intervention, because the calmer and more structured the setting was, the greater the improvement in task engagement that was noticed. Because the brushing occurred consecutively from one setting to the next, it is difficult to assess if the increase in improvements was due to the setting itself or to the effects of increased duration of the intervention. Confounding factors, including the boy's father leaving town and a friend leaving school with kidney stones, seemed to negatively impact on the child's results. ${ }^{30}$

A case study published in a non-peer-reviewed journal charted the results of intervention in two boys. One boy, in his third grade at school, showed some fluctuating results with attending to tasks after receiving a sensory diet for two weeks. Subsequently, the Wilbarger deep-pressure-brushing protocol was introduced during school hours, and it was reported that he met his intervention goals after 6 weeks. It was difficult to assess the involvement of the Wilbarger protocol intervention in the other boy. Precise treatment fidelity data were not reported. ${ }^{31}$ An additional master's thesis exists at the University of Puget Sound, ${ }^{32}$ which was contacted; however, we were unable to access this thesis because it was missing at the time of the review.

\section{Perceived efficacy}

Some of these studies also explored the effectiveness of the Wilbarger protocol from the perspective of the parent, caregiver, or treating occupational therapist. One study found that four out of five parents administering the Wilbarger protocol over a 6-week period perceived it to be effective for their child. ${ }^{28}$ A randomized controlled study showed that parents who administered the Wilbarger intervention perceived a significantly greater reduction in severity of challenging behavior and greater contribution to their child's daily life when compared with parents administering a behavioral intervention. There was no difference between these two groups for perceptions regarding reducing their child's frequency of challenging behavior. ${ }^{26}$

In a qualitative study by Segal and Beyer, ${ }^{33}$ perceived efficacy was a theme that emerged as an enabler or barrier to parental adherence with the Wilbarger protocol. Quotes from the parents on their perceptions were reported, and while treatment efficacy was not an aim of the study, no conclusions were drawn by the authors with regard to the effect of treatment. However, the majority of parents who suggested some initial positive changes in their child were unsure if the changes were due to the Wilbarger protocol, their child receiving multiple interventions, or coincidence. One parent reported that the effects wore off quickly, suggesting no long-term effects. ${ }^{33}$ Finally, Sudore reported her master's thesis in 2001, and described a random sample survey of special interest group members from the American Occupational Therapy Association. She showed that, of the 47 respondents, 15 thought the Wilbarger protocol was very effective, 29 somewhat effective, one somewhat ineffective, and two ineffective. ${ }^{18}$

\section{Limitations}

The results of the four studies included in this review had poor external validity. External validity refers to the extent to which the findings of a study sample can be generalized to the study population that is specified in the research question. ${ }^{34}$ The small sample sizes in all four studies meant that statistical significance could not be calculated, and therefore the findings were unable to be generalized to the wider population. Additionally, differences in outcome measures, treatment fidelity, and participant characteristics between the studies meant that collating of data was unable to be achieved to strengthen the results.

Several threats to internal validity exist. Internal validity refers to how precisely a research design answers the research question, and the level of confidence that the results are due to a causal relationship between independent and dependent variables. ${ }^{34}$ Selection bias was present in all studies because of the use of convenience sampling, as well as inclusion of participants who were amenable and likely to do well on the intervention. In one instance, it was stated that the participants were selected on the basis that they would benefit from the Wilbarger protocol due to previous positive responses to somatosensory input. ${ }^{25}$ In addition, the investigators were not blinded to the selection process used for all four studies. 
Other threats to internal validity were history and maturation. In all studies, the participants were receiving occupational therapy or behavioral therapy as a part of their usual therapy program. The studies were unable to eliminate the effects of maturation with such small sample sizes and a lack of control groups. Kimball et al attempted to eliminate the effects of maturation by conducting salivary cortisol measures immediately before and 15 minutes after a single application of the Wilbarger protocol, but did not describe any confounding factors that may have affected the cortisol changes. ${ }^{15}$ It is unclear whether the 15 minutes of neutral time following the single intervention caused the posttest change in cortisol levels or whether the change was attributed to the intervention itself.

Intervention bias existed in all studies. Contamination was a factor in the study reported by Benson et al because the control participant received an intervention quite similar to that of the participant receiving the Wilbarger protocol. ${ }^{25}$ Cointervention bias was present in all four studies. It is unclear if the long-term effects of improvement in the study by Stagnitti et $\mathrm{al}^{24}$ or the study by Davis et $\mathrm{al}^{16}$ were due to the Wilbarger protocol or other interventions. In the study by Kimball et al, it is unclear if the participants received treatment from different therapists, which could have biased the results. ${ }^{15}$ Additional sources of bias that could affect the internal validity of these studies include dropouts not being reported, instrumentation bias in the studies using observation without formal and researched tools, and experimenter bias, given that the allocation of intervention was not concealed from the examiners.

There are several factors that may explain why such limited research evidence exists on the Wilbarger protocol as prescribed. The Wilbarger protocol is an onerous program for caregivers and occupational therapists to administer. In a study by Segal and Beyer, it was found that parental adherence to the protocol was low. ${ }^{33}$ The factors that prevented faithful adherence to treatment on the part of parents included a negative response by their child to brushing, whether the parents could observe immediate effects of the intervention, and whether the parents could integrate the frequency of the protocol into their daily schedules. ${ }^{33}$ Additionally, the Wilbarger protocol is usually used in many different variations ${ }^{15}$ and in conjunction with a variety of sensory processing techniques.

This systematic review included only peer-reviewed evidence, so some publication bias may exist. Theses and dissertations testing the effectiveness of the Wilbarger protocol do exist. The authors of these studies are strongly encouraged to publish in peer-reviewed journals to contribute to and strengthen the current body of knowledge. Language bias should also be mentioned because English language studies were the only publications included in this review. Although an extensive search strategy was conducted, it is acknowledged by the authors that studies relevant to the research question may have been missed.

\section{Conclusion}

There is emerging evidence of the effectiveness of the Wilbarger protocol with children with regard to modulating cortisol levels, improving behavior, and increasing school and social participation. However, due to the paucity of studies and their low quality, the evidence for or against is limited.

Our systematic review highlights that the best available studies to test the effectiveness of the Wilbarger protocol with children is level IV (quantitative, case series with pretest/posttest outcomes) on the evidence hierarchy recommended by the Australian National Health and Medical Research Council. This is the lowest level of evidence. Whilst the recommendation of the Australian National Health and Medical Research Council is that the "body of evidence is weak and recommendations must be applied with caution,"22 it should be acknowledged that this is due to the small number of studies available and their low quality. Clinical decisionmaking should continue to be based on a thorough process that includes assessment, clinical reasoning, expertise, monitoring, and evaluation, with clear outcome measures and client-centered practice, until future high quality research has been produced.

The effectiveness of the Wilbarger protocol with children is a poorly researched area. Future research should include higher level study designs, such as randomized controlled trials and quasi-experimental protocols, which should include use of outcome measures that are standardized, well researched, and contain sound psychometric properties, larger and homogenous samples, and controls for cointervention. Future studies should also collect precise treatment fidelity data to advise of the exact implementation of the Wilbarger protocol being studied. High quality studies on the Wilbarger protocol as it is intended to be prescribed are crucial; however, data on the variations commonly used in practice may also be beneficial.

\section{Disclosure}

The authors report no conflicts of interest in this work.

\section{References}

1. Schaaf RC, Davies PL. Evolution of the sensory integration frame of reference. Am J Occup Ther. 2010;64(3):363-367. 
2. Arbesman M, Lieberman D. Methodology for the systematic reviews of occupational therapy for children and adolescents with difficulty processing and integrating sensory information. Am J Occup Ther. 2010;64(3): 368-374.

3. Parham LD, Cohn ES, Spitzer S, et al. Fidelity in sensory integration intervention research. Am J Occup Ther. 2007;61(2):216-227.

4. Zimmer M, Desch L. Policy statement: sensory integration therapies for children with developmental and behavioral disorders. Pediatrics. 2012; 129(6):1186-1189.

5. Davies PL, Gavin WJ. Validating the diagnosis of sensory processing disorders using EEG technology. Am J Occup Ther. 2007;61(2): 176-189.

6. Pollock N. Sensory integration: a review of the current state of the evidence. Occup Ther Now. 2009;11(5):6-10.

7. White BP, Mulligan S, Merrill K, Wright J. An examination of the relationships between motor and process skills and scores on the Sensory Profile. Am J Occup Ther. 2007;61(2):154-160.

8. Miller LJ, Anzalone ME, Lane SJ, Cermak SA, Osten ET. Concept evolution in sensory integration: a proposed nosology for diagnosis Am J Occup Ther. 2007;61(2):135-140.

9. Miller LJ, Lane SJ. Toward a consensus in terminology in sensory integration theory and practice: part 1: taxonomy of neurophysiological processes. Am J Occup Ther Sensory Integration Special Interest Section Quarterly. 2000;23(1):1-4.

10. Ahn RR, Miller LJ, Milberger S, McIntosh DN. Prevalence of parents' perceptions of sensory processing disorders among kindergarten children. Am J Occup Ther. 2004;58(3):287-293.

11. Wilbarger P, Wilbarger JL. Sensory Defensiveness in Children aged 2-12: An Intervention Guide for Parents and Other Caretakers. Santa Barbara, CA: Avanti Educational Programs; 1991.

12. Reynolds S, Lane SJ. Sensory overresponsivity and anxiety in children with ADHD. Am J Occup Ther. 2009;63(4):433-440.

13. Ben-Sasson A, Carter A, Briggs-Gowan M. Sensory over-responsivity in elementary school: prevalence and social-emotional correlates. J Abnorm Child Psychol. 2009;37(5):705-716.

14. Engel-Yeger B. The applicability of the short sensory profile for screening sensory processing disorders among Israeli children. Int $J$ Rehabil Res. 2010;33(4):311-318.

15. Kimball JG, Lynch KM, Stewart KC, Williams NE, Thomas MA, Atwood KD. Using salivary cortisol to measure the effects of a Wilbarger protocol-based procedure on sympathetic arousal: a pilot study. Am J Occup Ther. 2007;61(4):406-413.

16. Davis TN, Durand S, Chan JM. The effects of a brushing procedure on stereotypical behavior. Res Autism Spectr Disord. 2011;5(3): 1053-1058.

17. Foss A, Swinth Y, McGruder J, Tomlin G. Sensory modulation dysfunction and the Wilbarger protocol: an evidence review. OT Practice. 2003;8(12):1-7.

18. Sudore K. Tactile defensiveness and the Wilbarger brushing protocol in system management. Unpublished master's thesis. Buffalo, NY: D’Youville College; 2001.

19. Wilbarger JL, Wilbarger PL. Sensory Defensiveness: A Comprehensive Treatment Approach. Panorama City, CA: Avanti Educational Programs; 2007.

20. Polatajko HJ, Cantin N. Exploring the effectiveness of occupational therapy interventions, other than the sensory integration approach, with children and adolescents experiencing difficulty processing and integrating sensory information. Am J Occup Ther. 2010;64(3):415-429.
21. Law M, Stewart D, Pollock N, Letts L, Bosch J, Westmorland M. Critical review form for quantitative studies, 1998. Available from: http://www. srs-mcmaster.ca/Portals/20/pdf/ebp/quanreview.pdf. Accessed March 15, 2012.

22. National Health and Medical Research Council. NHMRC levels of evidence and grades for recommendations for developers of guidelines, 2009. Available from: http://www.nhmrc.gov.au/_files_nhmrc/file/ guidelines/evidence_statement_form.pdf. Accessed May 20, 2012.

23. Moher D, Liberati A, Tetzlaff J, Altman DG. Preferred reporting items for systematic reviews and meta-analyses: the PRISMA statement. PLoS Med. 2009;6(7):1-6.

24. Stagnitti K, Raison P, Ryan P. Sensory defensiveness syndrome: a paediatric perspective and case study. Aust Occup Ther J. 1999;46(4): 175-187.

25. Benson JD, Beeman E, Smitsky D, Provident I. The deep pressure and proprioceptive technique (dppt) versus nonspecific child-guided brushing: a case study. J Occup Ther Sch and Early Interv. 2011;4(3/4): 204-214.

26. Mora L, Chapparo C. The impact of the sensory protocol on the functional behavioural responses of children with intellectual disability, sensory defensiveness and behaviour support needs, 2008 Available from: http://www.asid.asn.au/Portals/0/Conferences/43rd\%20 Melbourne/Mora\%20Impact\%20of\%20the\%20Sensory\%20Protocol. pdf. Accessed September 7, 2012.

27. Chapparo C, Mora L. Use of a sensory protocol by parents of children with intellectual disability and sensory defensiveness to achieve functional and behavioural goals: a randomised controlled study. 24th National Conference and Exhibition of Occupational Therapy Australia; 2011 June 29-July 1; Gold Coast, Australia. Aust Occup Ther J. 2011;58:59.

28. Bhopti A. Examining the Wilbarger's deep pressure and proprioceptive technique for treating children with sensory defensiveness using a multiple single case study approach. 24th National Conference and Exhibition of Occupational Therapy Australia, ; 2011 June 29-July 1; Gold Coast, Australia. Aust Occup Ther J. 2011;58:134.

29. Zbytniewski R. The effects of sensory integration's Wilbarger technique on engaging behaviors [master's thesis]. Bay Shore, NY: Touro College; 2002.

30. Little TA. The effects of the Wilbarger protocol on task engagement in a child with Asperger's syndrome [doctoral dissertation]. Cookeville, TN: Tennessee Technological University; 2006.

31. Clark GF, Ward S. Charting results: evaluating progress of children with sensory processing disorder. OT Practice. 1999;4(10):12-16.

32. Snyder-Stonebraker D. The effects of a Wilbarger-based brushing protocol: a single subject design [master's thesis]. Tacoma, WA: University of Puget Sound; 2001.

33. Segal R, Beyer C. Integration and application of a home treatment program: a study of parents and occupational therapists. Am J Occup Ther. 2006;60(5):500-510.

34. DePoy E, Gitlin LN. Introduction to Research: Understanding and Applying Multiple Strategies. 4th ed. St Louis, MO: Elsevier Mosby; 2011.
Pediatric Health, Medicine and Therapeutics

\section{Publish your work in this journal}

Pediatric Health, Medicine and Therapeutics is an international, peerreviewed, open access journal publishing original research, reports, editorials, reviews and commentaries. All aspects of health maintenance preventative measures and disease treatment interventions are addressed within the journal. Practitioners from all disciplines are invited to submit

\section{Dovepress}

their work as well as healthcare researchers and patient support groups. The manuscript management system is completely online and includes a very quick and fair peer-review system. Visit http://www.dovepress.com/ testimonials.php to read real quotes from published authors. 